\title{
Nivel de razonamiento en la comprensión de gráficos estadísticos en estudiantes universitarios
}

\section{Level of reasoning in the understanding of statistical graphics in college students}

\section{Nível de razoamento na compreensão de gráficos estatísticas em estudantes universitários}

\author{
Pastor Ramírez-Leal ${ }^{1}$, César Augusto Hernández-Suárez², Raúl Prada-Núñez ${ }^{3}$
}

Forma de citar: P. Ramírez-Leal, C. A. Hernández-Suárez, R. Prada-Núñez, "Nivel de razonamiento en la comprensión de gráficos estadísticos en estudiantes universitarios", Respuestas, vol. 21, no. 2, pp. 13-23, 2016.

Recibido:

Diciembre 18 de 2015

Aceptado:

Marzo 14 de 2016

'Magister en Educación

Matemática

pastorramirez@ufps.edu.co Orcid: 0000-0003-3469-5325

Universidad Francisco de Paula

Santander

Cúcuta - Colombia

${ }^{2}$ Magister en Educación

Matemática

cesaraugusto@ufps.edu.co Orcid: 0000-0002-7974-5560 Universidad Francisco de Paula Santander Cúcuta-Colombia

${ }^{3}$ Magister en Educación Matemática raulprada@ufps.edu.co Orcid: 0000-0001-6145-1786 Universidad Francisco de Paula Santander Cúcuta - Colombia

\section{Resumen}

Antecedentes: El parámetro escogido para identificar el nivel de comprensión de gráficas estadísticas que poseen los estudiantes universitarios es el modelo M3ST (Middle School Stundents Statistical Thinking, por sus siglas en inglés, o, lo que es lo mismo, Pensamiento Estadístico en Estudiantes de Secundaria) propuesto por Langrall y Mooney (2002). Objetivo: La investigación que reporta este artículo ha tenido como propósito identificar el nivel de comprensión de gráficas estadísticas que poseen los estudiantes de la carrera de Administración de Empresas de la Universidad Francisco de Paula Santander (UFPS). Métodos: Con este fin se aplicó a una muestra de 111 estudiantes un cuestionario diseñado para evaluar dos aspectos del pensamiento estadístico en la comprensión de gráficas, a saber: descripción y representación de datos. Resultados: Del análisis de los resultados se obtuvo que la mayoría de los estudiantes se ubica en el nivel idiosincrásico. Conclusión: Lo cual significa que tales estudiantes presentan dificultades para distinguir los elementos de una gráfica, para establecer relaciones entre los elementos contenidos en ella y para justificar apropiadamente sus respuestas.

Palabras clave: Estudiantes universitarios, gráficas, organización y representación de datos, pensamiento estadístico.

\begin{abstract}
Background: The parameter chosen to identify the level of understanding of statistical graphs which college students have is the M3ST model (Middle School Students Statistical Thinking) proposed by Langrall and Mooney (2002). Objective: The research reported in this article has been aimed to identify the level of understanding of statistical graphs by students of the business administration program at the Francisco de Paula Santander University (UFPS). Methods: A questionnaire designed to evaluate two aspects of statistical thinking in graphs understanding, namely: description and data representation was applied to a sample of 111 students Results: The analysis of the results showed that most of the students are at the idiosyncratic level. Conclusion: This means that the group of students have difficulty distinguishing the elements of a graph, to establish relationships between the elements contained on it and to properly justify their answers.
\end{abstract}

Keywords: College students, graphs, organizing and representing data, statistical thinking. 
No. 2

Jul - Dic 2016

ISSN 0122-820X

E-ISSN 2422-5053

PP: 13-23

\section{Resumo}

Antecedentes: O parâmetro escolhido para identificar o nível de compreensão de figuras estatísticas que possuem os alunos universitários é o modelo M3ST (Middle School Stundents Statistical Thinking, por sua sigla em inglês, ou o que é o mesmo, Pensamento Estatístico em Alunos de Secundaria) proposto por Langrall e Mooney (2002). Objetivo: O propósito deste artigo foi reportar a pesquisa que teve como fim identificar o nível de compreensão de figuras estatísticas que possuem os alunos do curso de Administração de Empresas da Universidad Francisco de Paula Santander (UFPS). Métodos: Aplicou-se a uma amostra de cento e onze (111) estudantes, um questionário para avaliar dois aspectos do pensamento estatístico na compreensão de figuras: descrição e representação de dados. Resultados: Da análise dos resultados se percebeu que a maioria dos alunos se encontra no nível idiossincrásico. Conclusão: Os alunos apresentam dificuldades para distinguir os elementos de uma figura, assim como de estabelecer relações entre os elementos contidos nela e justificar apropriadamente suas respostas, o que corresponde a um nível idiossincrásico.

Palavras-chave: Estudantes universitários, gráficos, organização e representação de dados, pensamento estatístico.

\section{Introducción}

Comúnmente se considera que leer datos en una gráfica es una actividad simple y directa, pues se trata de un sistema de representación que facilita la comprensión de elementos que así expuestos resultan mucho más inteligibles. Sin embargo, las gráficas pueden tener distintos rangos de complejidad y de ellas se puede obtener información de distinta jerarquía de acuerdo con la capacidad de desciframiento e interpretación que tenga quien la consulta. Con frecuencia nos encontramos en el mundo contemporáneo con que distintos grupos sociales, políticos o económicos traducen los mismos datos o cifras de manera distinta según convenga a sus propios intereses, y así los muestran. Es por ello que un aspecto importante de la educación actual consiste en enseñar a "leer" estadísticas, de modo que, al igual que ocurre ante determinadas prácticas discursivas persuasivas, los ciudadanos estén capacitados para la crítica y el análisis y no al consumo inmediato y acrítico de ciertas formas de interpretación de la realidad que se provee a través de los medios de comunicación (tradicionales o electrónicos) y que sirven a determinados

propósitos. A este respecto se pronuncian [1] cuando afirman que "además de por su presencia en los medios de comunicación e Internet, el aprendizaje de las tablas y gráficos estadísticos es importante, por otros muchos motivos". Por un lado, son un potente instrumento para comunicar información y para resumirla en forma eficiente [2], [3], por su parte, hablan de la transmumeración como uno de los modos esenciales de razonamiento estadístico, que consiste en obtener una nueva información, que no estaba disponible en un conjunto de datos al cambiar de un sistema de representación a otro.

Si bien comprender y usar gráficas es una parte clave involucrada en el desarrollo del pensamiento estadístico [4] es igualmente cierto que se precisa de un determinado y necesario entrenamiento, tanto en la formación académica profesional como en la del ciudadano crítico ante la realidad. La lectura correcta de gráficos, de hecho, ha sido muy investigada en educación estadística, definiéndose diversos niveles en su comprensión [5]. Pero en las tareas didácticas se requiere no solo mostrar los distintos modos de graficar datos sino 
además explorar las dificultades que tienen los estudiantes en la comprensión de gráficas a fin de ayudarlos a entenderlas y a mejorar su lectura e interpretación [6]. En opinión [7], por otra parte, "muchos errores en la construcción del gráfico o en su correcta lectura e interpretación estarán ligados a falta de comprensión de algunos de los objetos estadísticos y sus relaciones que subyacen en el gráfico".

Desde una perspectiva específicamente situada, el perfil profesional de un egresado universitario, más concretamente, de la carrera de Administración de Empresas, supone la competencia para tomar continuamente decisiones a partir de la revisión de los distintos tipos de datos e informaciones que se generan en la actual sociedad del conocimiento. En consecuencia, todo graduado (y antes, cualquier estudiante universitario) debe poseer y desarrollar la habilidad de obtener, analizar, reducir, interpretar e incluso presentar información a través de una inmensa variedad de formatos textuales, iconográficos, simbólicos y gráficos.

Entre estos distintos modos de ofrecimiento y apropiación de información se encuentran, como se ha dicho, la elaboración y lectura de graficas estadísticas. Esta es una de las razones por las cuales el programa académico de la carrera de Administración de Empresas de la Universidad Francisco de Paula Santander (UFPS) incluye en su programa académico la asignatura Estadística Descriptiva, que forma parte del eje de formación del área básica de Matemáticas y se imparte durante el segundo semestre.

El propósito general de la asignatura consiste en desarrollar en el estudiante las competencias necesarias para la investigación mediante el empleo de métodos estadísticos. Estas competencias, desde luego, se orientan al campo de la Administración, con la necesaria implicación, como no puede ser de otra manera, del contexto sociológico propio de cada realidad en las que el profesional se desenvuelve. Entre los objetivos más concretos de la materia se encuentra el desarrollo de la capacidad de los estudiantes para la interpretación y análisis de datos, tablas y gráficos; el conocimiento de las características, usos, ventajas y desventajas de las mediadas de posición y dispersión, lo mismo que su cálculo, interpretación y explicación; la asignatura también se propone, por último, el cálculo e interpretación de estadísticos probabilísticos útiles para el proceso de toma de decisiones. Toda esta información será en última instancia necesaria para descifrar el nivel de confianza que ofrecen las representaciones gráficas [8].

En relación con lo anterior, el objeto de la investigación que se reporta en este artículo consistió en identificar el nivel de comprensión de gráficas estadísticas que poseen los estudiantes de la carrera de Administración de Empresas de la Universidad Francisco de Paula Santander (UFPS) en la ciudad de Cúcuta (Colombia) a fin de, en una fase posterior del trabajo, ofrecer herramientas que permitan mejorar este aspecto de su formación. El parámetro escogido para esta medición ha sido el modelo M3ST (Middle School Stundents Statistical Thinking, por sus siglas en inglés, o, lo que es lo mismo, Pensamiento Estadístico en Estudiantes de Secundaria) propuesto por [9]. La elección del modelo tiene que ver con su adecuación probada por [10] a un contexto semejante al que aquí se explora.

\section{Materiales y métodos}

\subsection{Materiales}

Esta investigación es de carácter descriptivo por cuanto busca caracterizar una población de individuos. Este tipo de metodología emplea mediciones que incluyen porcentajes de aciertos o desaciertos o niveles de complejidad alcanzados durante la realización de determinadas actividades. La población
Jul - Dic 2016

ISSN 0122-820X

E-ISSN 2422-5053

PP: $13-23$ 
No. 2

Jul - Dic 2016 ISSN 0122-820X E-ISSN 2422-5053

PP: 13-23 objeto de este estudio estuvo constituida por los estudiantes matriculados en la asignatura Estadística Descriptiva, que corresponde al segundo semestre, turno diurno, de la carrera Administración de Empresas de la Facultad de Ciencias Empresariales de la Universidad Francisco de Paula Santander. E, distribuidos de la siguiente manera: curso A: 50 estudiantes, curso B: 47 estudiantes, curso C: 46 estudiantes y curso D: 46 estudiantes, para un total de 189 sujetos.

Para la investigación se consideraron dos procesos estadísticos: descripción y representación de datos. Para el primero de estos dos, es decir, para la descripción de datos, se establecieron tres niveles de complejidad: un nivel elemental, enfocado sobre la lectura de datos de la gráfica ('leer la gráfica'); un nivel intermedio, que involucra la interpolación y extracción de información de los datos mostrados en la gráfica ('leer dentro de la gráfica') y un nivel general, que supone la extrapolación de los datos y la interpretación de las relaciones identificadas en la gráfica ('leer más allá de la gráfica') [9],[11].

\subsection{Métodos}

Para la determinación del tamaño de la muestra según las características de la población se aplicó el muestreo aleatorio en dos fases. En primer lugar, se utilizó el muestreo aleatorio simple para poblaciones finitas con los siguientes parámetros: tamaño de la población: $\mathrm{N}=189$; nivel de confianza 95\%: $Z=1,96$; margen de error: $6 \%, p=0,5$. Esto arrojó un tamaño de muestra (n) de 111 estudiantes. Para determinar el tamaño de la muestra según la población de cada grupo se aplicó el muestreo aleatorio estratificado con afijación proporcional, quedando estructurada de la siguiente manera: 30 estudiantes del curso A, 27 estudiantes del curso B, 27 estudiantes del curso C y 27 estudiantes del curso D.
Se elaboró un cuestionario de dos preguntas de respuesta abierta con el fin de evaluar los dos aspectos del pensamiento estadístico señalados (descripción y representación de datos) en la comprensión de gráficos y sus respectivos componentes. La demostración de competencias en distinto grado de complejidad se analiza en cada respuesta ubicándola dentro de los niveles propuestos por el modelo M3ST [9].

Las preguntas se elaboraron considerando los cuatro niveles propuestos por el modelo M3ST, los cuales permiten identificar dos subprocesos en la comprensión de gráficas estadísticos, modificándolos de acuerdo con el propósito de esta investigación, que se dirige hacia la identificación de las dificultades que presentan los estudiantes de segundo semestre de Administración de Empresas de la UFPS en la comprensión de gráficas estadísticas. El instrumento ya ha sido validado por otros estudios como [12], sin embargo, considerando la adaptación realizada, el cuestionario fue sometido a validación por medio de la técnica del juicio de expertos obteniendo resultados satisfactorios.

El proceso de descripción de datos y sus componentes fueron medidos de la siguiente manera:

Con el diagrama de frecuencias y la pregunta 1A, inciso a (¿Cuántos días fueron observados con demanda de 34 unidades?) se evaluó la capacidad que tienen los estudiantes para 'leer datos de una gráfica'. Esta 'lectura' implica que los estudiantes debían ser capaces de reconocer las unidades de medida de cada eje, en este caso, reconocer que el eje x mide la cantidad de unidades demandadas, mientras el eje $y$ refiere las frecuencias de dichas cantidades demandadas diariamente; para 'leer un dato' los estudiantes debían asimismo ser capaces de relacionar la cantidad de unidades demandadas con su frecuencia diaria. 
Mediante la pregunta 1A, inciso b) (¿Cuántos días fueron observados para analizar la demanda del producto?) se exploró la habilidad que tienen los estudiantes para 'leer datos dentro de una gráfica'. Responder correctamente la pregunta suponía ubicar los valores específicos de las cantidades demandadas en el eje $x$, asociarlas con los valores específicos de las frecuencias de los días en el eje $y$ y sumar ambas frecuencias.

Para responder a la pregunta $1 \mathrm{~A}$, inciso c) (Suma las cantidades demandadas en 60 días de observación donde hubo mayor demanda del producto) los estudiantes debían identificar los valores de los datos específicos y sumarlos, en este caso, identificar las cantidades de mayor demanda del producto en el eje $x$ asociarlas con las frecuencias de días en el eje $y$ sumar una a una dichas frecuencias hasta completar los 60 días.

Las respuestas permitieron ubicar a los estudiantes en las categorías propuestas por el Modelo M3ST desarrollado por [9] tal y como se muestra la Tabla I.

Tabla I. Niveles propuestos por el modelo M3ST

\begin{tabular}{|c|c|}
\hline $\begin{array}{l}\text { Nivel 1. Idiosincrásico } \\
\text { D.1.1 Demuestra poco conocimiento de las } \\
\text { características exhibidas de una tabla, cuadro o } \\
\text { representación gráfica. } \\
\text { D.1.2 Malinterpreta o no identifica las unidades de } \\
\text { los valores de los datos. }\end{array}$ & $\begin{array}{l}\text { Nivel 2. Transicional } \\
\text { D.2.1 Demuestra algo de conocimiento de las } \\
\text { características exhibidas de una tabla, cuadro o } \\
\text { representación gráfica. } \\
\text { D.2.2 Identifica las unidades de los valores de los datos } \\
\text { incompletamente. }\end{array}$ \\
\hline $\begin{array}{l}\text { Nivel 3. Cuantitativo } \\
\text { D.3.1 Demuestra conocimiento completo de las } \\
\text { características exhibidas de una tabla, cuadro o } \\
\text { representación gráfica. } \\
\text { D.3.2 Identifica las unidades de los valores de datos } \\
\text { específicos. }\end{array}$ & $\begin{array}{l}\text { Nivel 4. Analítico } \\
\text { D.4.1 Demuestra conocimiento completo de las } \\
\text { características exhibidas de una tabla, cuadro o } \\
\text { representación gráfica incluyendo características } \\
\text { irrelevantes o superficiales. } \\
\text { D.4.2 Identifica las unidades de los valores de datos } \\
\text { generales. }\end{array}$ \\
\hline
\end{tabular}

Fuente: [9]

La ubicación dentro de cada categoría se realizó analizando las respuestas numéricas y verbales dadas por los estudiantes. Asimismo se identificaron dos subprocesos en la comprensión de gráficas, uno analítico: D.1 Mostrar conocimiento para las características exhibidas; y otro cuantitativo: D.2 Identificar las unidades de los valores de los datos. Para categorizar el pensamiento de un estudiante se consideraron los dos procesos.

Como se ha señalado con anterioridad, la pregunta 2 se refirió a la organización y representación de datos. Para contestarla el estudiante debía poseer habilidades de conteo y ordenación de datos, así como para incorporarlos en una gráfica de barras.

\section{Resultados y análisis}

Las siguientes tablas resumen las respuestas de los estudiantes y su ubicación en los cuatro niveles del modelo M3ST.
La Tabla II resume las frecuencias y porcentajes de las respuestas de los estudiantes de cada grupo y su categorización para el nivel idiosincrásico según el modelo M3ST. De acuerdo con tales respuestas el grupo B, que obtuvo un porcentaje del 53.7, resulta el grupo más idiosincrásico, seguido del grupo $\mathrm{D}$, con un $51.9 \%$, luego por el grupo $\mathrm{A}$, con un $48.32 \%$ y finalmente por el C, con un $47.22 \%$. El nivel idiosincrásico obtenido en promedio es del $50.23 \%$, por lo cual se sitúa como el nivel mayoritario en este estudio. Estos valores reflejan que el grupo presenta serias dificultades para comprender, interpretar e identificar las unidades y valores de los gráficos y darle una lectura con significado real. Entre las respuestas verbales en este nivel resaltan argumentos de los estudiantes que hacen referencia a la ausencia de una idea clara sobre la respuesta como "no sé" o respuestas muy alejadas de la correcta como "39 días fueron utilizados para la semana del producto". 
Tabla II. Respuestas registradas para el nivel idiosincrásico

\begin{tabular}{|c|c|c|c|c|c|c|c|c|c|c|c|}
\hline \multicolumn{2}{|c|}{ Grupo } & \multicolumn{2}{|c|}{ Pregunta 1Aa } & \multicolumn{2}{|c|}{ Pregunta 1Ab } & \multicolumn{2}{|c|}{ Pregunta 1Ac } & \multicolumn{2}{|c|}{ Pregunta 2A } & \multicolumn{2}{|c|}{ Total Resp } \\
\hline & & Idiosin. & Otros & Idiosin. & Otros & Idiosin. & Otros & Idiosin. & Otros & Idiosin. & Otros \\
\hline \multirow[t]{2}{*}{$\mathbf{A}$} & f & 4 & 26 & 28 & 2 & 23 & 7 & 3 & 27 & 58 & 62 \\
\hline & $\%$ & 3,60 & 23,42 & 25,23 & 1,80 & 20,72 & 6,31 & 2,70 & 24,32 & 48,33 & 51,67 \\
\hline \multirow[t]{2}{*}{ B } & f & 2 & 25 & 25 & 2 & 22 & 5 & 9 & 18 & 58 & 50 \\
\hline & $\%$ & 1,80 & 22,52 & 22,52 & 1,80 & 19,82 & 4,50 & 8,11 & 16,22 & 53,70 & 46,30 \\
\hline \multirow[t]{2}{*}{$\mathrm{C}$} & f & 5 & 22 & 25 & 2 & 17 & 10 & 4 & 23 & 51 & 57 \\
\hline & $\%$ & 4,50 & 19,82 & 22,52 & 1,80 & 15,32 & 9,01 & 3,60 & 20,72 & 47,22 & 52,78 \\
\hline \multirow[t]{2}{*}{ D } & f & 4 & 23 & 26 & 1 & 21 & 6 & 5 & 22 & 56 & 52 \\
\hline & $\%$ & 3,60 & 20,72 & 23,42 & 0,90 & 18,92 & 5,41 & 4,50 & 19,82 & 51,85 & 48,15 \\
\hline \multicolumn{2}{|c|}{ Total } & 15 & 96 & 104 & 7 & 83 & 28 & 21 & 90 & 223 & 221 \\
\hline & & 13,51 & 86,49 & 93,69 & 6,31 & 74,77 & 25,23 & 18,92 & 81,08 & 50,23 & 49,77 \\
\hline \multicolumn{2}{|c|}{ Total \% } & \multicolumn{2}{|c|}{100,00} & \multicolumn{2}{|c|}{100,00} & \multicolumn{2}{|c|}{100,00} & \multicolumn{2}{|c|}{100,00} & \multicolumn{2}{|c|}{100,00} \\
\hline \multicolumn{2}{|c|}{$\mathbf{n}$} & \multicolumn{2}{|c|}{111} & \multicolumn{2}{|c|}{111} & \multicolumn{2}{|c|}{111} & \multicolumn{2}{|c|}{111} & & \\
\hline
\end{tabular}

Fuente: Los Autores

En la Tabla II también se observa que la pregunta $1 \mathrm{~A}$, inciso b) alcanza un porcentaje del 93.69 y se convierte por tanto en la pregunta con más respuestas idiosincrásicas. La pregunta reza lo siguiente: ¿Cuántos días fueron observados para analizar la demanda del producto? Explique su respuesta. Por su intermedio se exploró la habilidad que tienen los estudiantes para 'leer datos dentro de una gráfica’. Los yerros consistieron en asociación establecida por los estudiantes entre el último de los ítems del eje $y$ la frecuencia de los días con el total de días observados para la demanda o la asociación de la altura de la barra de mayor frecuencia con el total de días observados para la demanda. La pregunta 1A, inciso c) obtuvo un porcentaje del 74.77, la pregunta $2 \mathrm{~A}$ un $18.92 \%$ y la pregunta $1 \mathrm{~A}$, inciso a) un $13.51 \%$.

La Tabla III refleja en frecuencias y porcentajes las respuestas los estudiantes de cada grupo y su categorización para el nivel Transicional según el modelo M3ST. Según las respuestas dadas se puede observar que el grupo A obtuvo un porcentaje del 25.83, resultando, por tanto, el grupo más transicional. Le sigue el grupo $\mathrm{C}$, con un $25 \%$, luego el grupo B con un $21.30 \%$ y el por último el grupo $\mathrm{D}$, con un $19.44 \%$. El nivel transicional obtuvo en promedio un porcentaje aproximado del $23 \%$, con lo cual se sitúa en el tercer lugar.

Tabla III. Respuestas registradas para el nivel transicional

\begin{tabular}{|c|c|c|c|c|c|c|c|c|c|c|c|}
\hline \multirow{2}{*}{\multicolumn{2}{|c|}{ Grupo }} & \multicolumn{2}{|c|}{ Pregunta 1Aa } & \multicolumn{2}{|c|}{ Pregunta 1Ab } & \multicolumn{2}{|c|}{ Pregunta 1Ac } & \multicolumn{2}{|c|}{ Pregunta 2A } & \multicolumn{2}{|c|}{ Total Resp } \\
\hline & & Transi. & Otros & Transi. & Otros & Transi. & Otros & Transi. & Otros & Transi. & Otros \\
\hline \multirow[t]{2}{*}{$\mathbf{A}$} & f & 0 & 30 & 2 & 28 & 7 & 23 & 22 & 8 & 31 & 89 \\
\hline & $\%$ & 0,00 & 27,03 & 1,80 & 25,23 & 6,31 & 20,72 & 19,82 & 7,21 & 25,83 & 74,17 \\
\hline \multirow[t]{2}{*}{ B } & f & 1 & 26 & 2 & 25 & 5 & 22 & 15 & 12 & 23 & 85 \\
\hline & $\%$ & 0,90 & 23,42 & 1,80 & 22,52 & 4,50 & 19,82 & 13,51 & 10,81 & 21,30 & 78,70 \\
\hline \multirow[t]{2}{*}{$\mathrm{C}$} & f & 1 & 26 & 0 & 27 & 10 & 17 & 16 & 11 & 27 & 81 \\
\hline & $\%$ & 0,90 & 23,42 & 0,00 & 24,32 & 9,01 & 15,32 & 14,41 & 9,91 & 25,00 & 75,00 \\
\hline \multirow[t]{2}{*}{ D } & f & 3 & 24 & 1 & 26 & 5 & 22 & 12 & 15 & 21 & 87 \\
\hline & $\%$ & 2,70 & 21,62 & 0,90 & 23,42 & 4,50 & 19,82 & 10,81 & 13,51 & 19,44 & 80,56 \\
\hline \multicolumn{2}{|c|}{ Total } & 5 & 106 & 5 & 106 & 27 & 84 & 65 & 46 & 102 & 342 \\
\hline & & 4,50 & 95,50 & 4,50 & 95,50 & 24,32 & 75,68 & 58,56 & 41,44 & 22,97 & 77,03 \\
\hline \multicolumn{2}{|c|}{ Total \% } & \multicolumn{2}{|c|}{100,00} & \multicolumn{2}{|c|}{100,00} & \multicolumn{2}{|c|}{100,00} & \multicolumn{2}{|c|}{100,00} & \multicolumn{2}{|c|}{100,00} \\
\hline \multicolumn{2}{|c|}{$\mathbf{n}$} & \multicolumn{2}{|c|}{111} & \multicolumn{2}{|c|}{111} & \multicolumn{2}{|c|}{111} & \multicolumn{2}{|c|}{111} & & \\
\hline
\end{tabular}

Fuente: Los Autores

También se puede observar en la Tabla III que la pregunta $2 \mathrm{~A}$, con un porcentaje del $58.56 \%$, es la pregunta con más respuestas transicionales dadas por los estudiantes. El enunciado de la pregunta es el siguiente: Complete la tabla de frecuencia que se presenta a continuación y bosqueje un diagrama de barras con los datos obtenidos.
Para contestarla, el estudiante debía poseer habilidades de conteo y organización de datos, así como para representarlos en una gráfica de barras.

Se observaron aproximaciones incipientes a la respuesta correcta, justificadas en desacertadas respuestas como "273 días". 
Los equívocos aparecieron cuando los estudiantes construyeron una gráfica parcialmente completa y representativa de los datos o completa y no representativa de los datos, cuando identificaron parcialmente los elementos de la gráfica, cuando realizaron un conteo adecuado de las frecuencias de los datos pero confundieron los ejes (en el eje $y$ ubicaron los puntos de aceptación y en el eje $x$ las frecuencias), no utilizaron etiquetas para identificar las variables expresadas en la gráfica o cuando, aun haciendo un conteo adecuado de las frecuencias de los datos e identificando correctamente los ejes, ubicaron las frecuencias en el eje $y$ y en el eje $\mathrm{x}$ los puntos. También erraron al confundir una gráfica de barras con un histograma. Se observa también en la Tabla III que la segunda pregunta con mayor concentración de respuestas de tipo transicional es la 1.A inciso c) con un porcentaje de $24.32 \%$.

Tabla IV. Respuestas registradas para el nivel cuantitativo

\begin{tabular}{|c|c|c|c|c|c|c|c|c|c|c|c|}
\hline \multicolumn{2}{|c|}{ Grupo } & \multicolumn{2}{|c|}{ Pregunta 1Aa } & \multicolumn{2}{|c|}{ Pregunta 1Ab } & \multicolumn{2}{|c|}{ Pregunta 1Ac } & \multicolumn{2}{|c|}{ Pregunta 2A } & \multicolumn{2}{|c|}{ Total Resp } \\
\hline & & Cuanti. & Otros & Cuanti. & Otros & Cuanti. & Otros & Cuanti. & Otros & Cuanti. & Otros \\
\hline \multirow[t]{2}{*}{$\mathbf{A}$} & f & 26 & 4 & 0 & 30 & 0 & 30 & 3 & 27 & 29 & 91 \\
\hline & $\%$ & 23,42 & 3,60 & 0,00 & 27,03 & 0,00 & 27,03 & 2,70 & 24,32 & 24,17 & 75,83 \\
\hline \multirow[t]{2}{*}{ B } & $\mathbf{f}$ & 24 & 3 & 0 & 27 & 0 & 27 & 2 & 25 & 26 & 82 \\
\hline & $\%$ & 21,62 & 2,70 & 0,00 & 24,32 & 0,00 & 24,32 & 1,80 & 22,52 & 24,07 & 75,93 \\
\hline \multirow[t]{2}{*}{$\mathrm{C}$} & f & 19 & 8 & 1 & 26 & 0 & 27 & 5 & 22 & 25 & 83 \\
\hline & $\%$ & 17,12 & 7,21 & 0,90 & 23,42 & 0,00 & 24,32 & 4,50 & 19,82 & 23,15 & 76,85 \\
\hline \multirow[t]{2}{*}{ D } & f & 20 & 7 & 0 & 27 & 0 & 27 & 9 & 18 & 29 & 79 \\
\hline & $\%$ & 18,02 & 6,31 & 0,00 & 24,32 & 0,00 & 24,32 & 8,11 & 16,22 & 26,85 & 73,15 \\
\hline \multicolumn{2}{|c|}{ Total } & 89 & 22 & 1 & 110 & 0 & 111 & 19 & 92 & 109 & 335 \\
\hline & & 80,18 & 19,82 & 0,90 & 99,10 & 0,00 & 100,00 & 17,12 & 82,88 & 24,55 & 75,45 \\
\hline \multicolumn{2}{|c|}{ Total \% } & \multicolumn{2}{|c|}{100,00} & \multicolumn{2}{|c|}{100,00} & \multicolumn{2}{|c|}{100,00} & \multicolumn{2}{|c|}{100,00} & \multicolumn{2}{|c|}{100,00} \\
\hline \multicolumn{2}{|c|}{ n } & \multicolumn{2}{|c|}{111} & \multicolumn{2}{|c|}{111} & \multicolumn{2}{|c|}{111} & \multicolumn{2}{|c|}{111} & & \\
\hline
\end{tabular}

Fuente: Los Autores

Asimismo se observa en la Tabla IV que la pregunta 1.A, inciso a) es, con un porcentaje del 80.18 , la que amalgama el mayor número de respuestas de índole cuantitativa. El planteamiento de la pregunta es el siguiente: ¿Cuántos días fueron observados con demanda de 34 unidades? Explique su respuesta. Mediante este ítem se evaluó la capacidad que tienen los estudiantes para 'leer datos de una gráfica', lo que implica poseer la capacidad de reconocer las unidades de medida de cada eje (en este caso, reconocer que el eje $x$ mide la cantidad de unidades demandadas, mientras el eje $y$ las frecuencias de dichas cantidades demandadas diariamente) y relacionar la cantidad de
Las preguntas 1.A inciso a) y 1.A inciso b) obtuvieron cada una un porcentaje de 4.5.

La Tabla IV muestra las frecuencias y porcentajes de las respuestas dadas por los estudiantes de cada grupo y su categorización dentro del nivel cuantitativo del modelo M3ST. En la Tabla se observa que el grupo D obtuvo un porcentaje del 26.85, con lo cual es el grupo que acumula más respuestas del tipo cuantitativo. Le siguen el grupo A, con un $24.17 \%$, luego el grupo B, con un $24,07 \%$ $\mathrm{y}$ en el último lugar el grupo $\mathrm{C}$, con un $23.15 \%$. En promedio, el nivel de respuestas de carácter cuantitativo obtuvo un porcentaje del 24.55, por lo cual se sitúa en el segundo lugar dentro de las respuestas dadas por los estudiantes. Las justificaciones de la respuesta en este nivel fueron acertadas, por ejemplo, en argumentos como: "se observaron 180 días para analizar a demanda del producto". unidades demandadas con su frecuencia diaria. En ello, en efecto, consiste 'leer un dato' de esta gráfica. Los errores cometidos por los estudiantes estuvieron en colocar la cantidad correcta sin justificar el porqué de la respuesta. También se observa en la Tabla III que la segunda pregunta con mayor índice de respuestas cuantitativas es la 2.A, con un porcentaje de 17.12. La pregunta 1.A inciso b) obtuvo un $0.90 \%$ y la pregunta $1 . \mathrm{A}$ inciso c) no fue evaluada cuantitativamente pues obtuvo un $0 \%$ de porcentaje.

E-ISSN 2422-5053

PP: 13-23
La Tabla V contiene las frecuencias y porcentajes de las respuestas de los 
No. 2

Jul - Dic 2016 ISSN 0122-820X E-ISSN 2422-5053

PP: 13-23 estudiantes de cada grupo y su categorización para el nivel analítico según el modelo M3ST. En ella se observa que el grupo C obtuvo un porcentaje del 4.63 y que, por consiguiente es el grupo que más respuestas de corte analítico acumula. Le siguen el grupo $\mathrm{D}$, con un $1.85 \%$, el grupo $\mathrm{A}$, con un $1.67 \%$ y el $\mathrm{B}$, con un $0.93 \%$. Las respuestas de tipo analítico alcanzan un porcentaje de solo $2.25 \%$, con lo cual se convierten en las menos empleadas por los estudiantes encuestados. Aunque poco evidentes, la justificación de la respuesta en este nivel goza de mayor profundidad y abstracción; por ejemplo, en una de las respuestas de los estudiantes se expresa que: "180 días, porque se suman los días que se examinó a demanda".

Tabla V. Respuestas registradas para el nivel analítico

\begin{tabular}{|c|c|c|c|c|c|c|c|c|c|c|c|}
\hline \multirow{2}{*}{\multicolumn{2}{|c|}{ Grupo }} & \multicolumn{2}{|c|}{ Pregunta 1Aa } & \multicolumn{2}{|c|}{ Pregunta 1Ab } & \multicolumn{2}{|c|}{ Pregunta 1Ac } & \multicolumn{2}{|c|}{ Pregunta 2A } & \multicolumn{2}{|c|}{ Total Resp } \\
\hline & & Analí. & Otros & Analí. & Otros & Analí. & Otros & Analí. & Otros & Analí. & Otros \\
\hline \multirow[t]{2}{*}{$\mathbf{A}$} & f & 0 & 30 & 0 & 30 & 0 & 30 & 2 & 28 & 2 & 118 \\
\hline & $\%$ & 0,00 & 27,03 & 0,00 & 27,03 & 0,00 & 27,03 & 1,80 & 25,23 & 1,67 & 98,33 \\
\hline \multirow[t]{2}{*}{ B } & f & 0 & 27 & 0 & 27 & 0 & 27 & 1 & 26 & 1 & 107 \\
\hline & $\%$ & 0,00 & 24,32 & 0,00 & 24,32 & 0,00 & 24,32 & 0,90 & 23,42 & 0,93 & 99,07 \\
\hline \multirow[t]{2}{*}{$\mathrm{C}$} & f & 2 & 25 & 1 & 26 & 0 & 27 & 2 & 25 & 5 & 103 \\
\hline & $\%$ & 1,80 & 22,52 & 0,90 & 23,42 & 0,00 & 24,32 & 1,80 & 22,52 & 4,63 & 95,37 \\
\hline \multirow[t]{2}{*}{ D } & f & 0 & 27 & 0 & 27 & 1 & 26 & 1 & 26 & 2 & 106 \\
\hline & $\%$ & 0,00 & 24,32 & 0,00 & 24,32 & 0,90 & 23,42 & 0,90 & 23,42 & 1,85 & 98,15 \\
\hline \multicolumn{2}{|c|}{ Total } & 2 & 109 & 1 & 110 & 1 & 110 & 6 & 105 & 10 & 434 \\
\hline & & 1,80 & 98,20 & 0,90 & 99,10 & 0,90 & 99,10 & 5,41 & 94,59 & 2,25 & 97,75 \\
\hline \multicolumn{2}{|c|}{ Total \% } & \multicolumn{2}{|c|}{100,00} & \multicolumn{2}{|c|}{100,00} & \multicolumn{2}{|c|}{100,00} & \multicolumn{2}{|c|}{100,00} & \multicolumn{2}{|c|}{100,00} \\
\hline \multicolumn{2}{|c|}{$\mathbf{n}$} & \multicolumn{2}{|c|}{111} & \multicolumn{2}{|c|}{111} & \multicolumn{2}{|c|}{111} & \multicolumn{2}{|c|}{111} & & \\
\hline
\end{tabular}

En esta Tabla también se evidencia que la pregunta $2 \mathrm{~A}$, con un porcentaje del $5.4 \%$, es la pregunta que aglutina una mayor proporción de contestaciones analíticas. Tal pregunta es la siguiente: Complete la tabla de frecuencia que se presenta a continuación y bosqueje un diagrama de barras con los datos obtenidos. Resulta obvio que el porcentaje de respuestas apropiadas es muy bajo y que el ideal sería que la mayoría de estudiantes estuviesen ubicados aquí. Lo mismo igual puede decirse para el resto de las preguntas, pues para la 1.A inciso a) el porcentaje fue de 1.8, y tanto para la pregunta 1.A, inciso b) como para la 1.A inciso c) el porcentaje fue de apenas 0.9.

La Figura 1 permite apreciar que el nivel predominante en el plano descriptivo es el idiosincrático, seguido del cuantitativo, luego del transicional y finalmente del analítico. Visualmente se observa que el nivel idiosincrático supera en cuantía a los otros niveles, lo cual da una idea de la capacidad reducida del grupo para comprender los elementos básicos en una lectura apropiada de los gráficos.

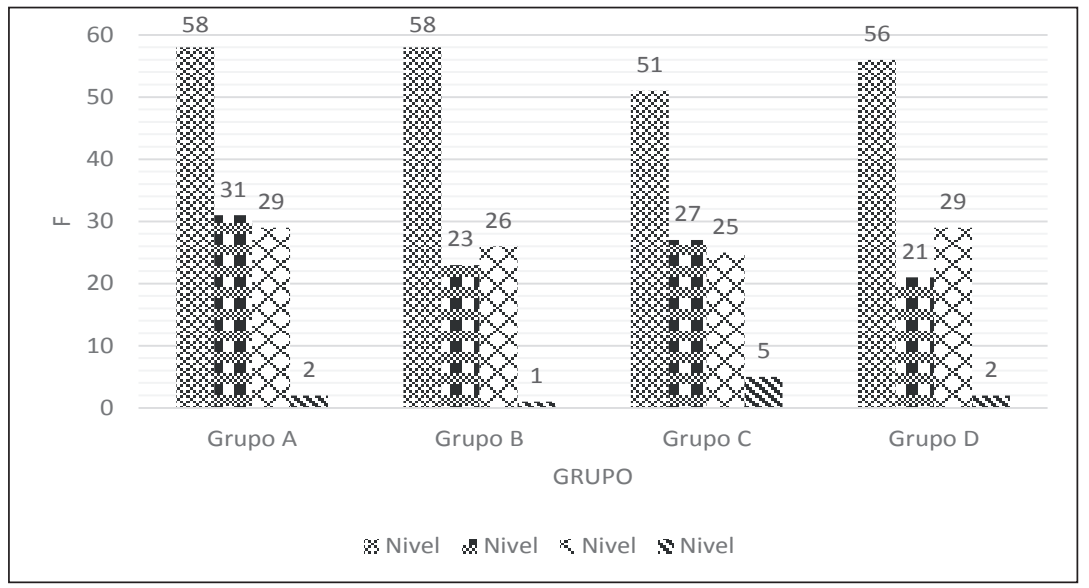

Figura 1. Relación de respuestas asociadas al nivel de comprensión Fuente: Los Autores 
En la Tabla VI se puede observar que aproximadamente la mitad de las respuestas dadas por los estudiantes a los problemas planteados se ubica en el nivel idiosincrático, es decir, los estudiantes demuestran escasa destreza para interpretar las características de las gráficas presentadas, lo que les dificultada su lectura y posterior interpretación. El nivel cuantitativo supera ligeramente al nivel transicional en poco más del 1\%, ubicándose en este nivel aproximadamente un $24 \%$ de las respuestas, en comparación con el 23\% del nivel transicional. Estos valores conducen a la interpretación de que tan sólo una cuarta parte de la muestra analizada tiene una idea referencial acerca de cómo leer e interpretar la información del gráfico. Aproximadamente el $2 \%$ de las respuestas analizadas se ubica en el nivel analítico, lo que indica que el grupo de estudiantes que son capaces de leer más allá de los datos presentados en el gráfico es mínimo.

Tabla VI. Distribución porcentual de respuestas por nivel de comprensión

\begin{tabular}{|c|c|c|c|c|c|c|c|}
\hline & \multicolumn{4}{|c|}{ Nivel } & \multirow{2}{*}{ Total } \\
\hline & & & Idiosincrático & Transicional & Cuantitativo & Analítico & \\
\hline \multirow{8}{*}{ Grupo } & \multirow[t]{2}{*}{ Grupo A } & $\mathrm{f}$ & 58 & 31 & 29 & 2 & 120 \\
\hline & & $\%$ & $13,1 \%$ & $7,0 \%$ & $6,5 \%$ & $0,5 \%$ & $27,0 \%$ \\
\hline & \multirow[t]{2}{*}{ Grupo B } & $\mathrm{f}$ & 58 & 23 & 26 & 1 & 108 \\
\hline & & $\%$ & $13,1 \%$ & $5,2 \%$ & $5,9 \%$ & $0,2 \%$ & $24,3 \%$ \\
\hline & \multirow[t]{2}{*}{ Grupo C } & $\mathrm{f}$ & 51 & 27 & 25 & 5 & 108 \\
\hline & & $\%$ & $11,5 \%$ & $6,1 \%$ & $5,6 \%$ & $1,1 \%$ & $24,3 \%$ \\
\hline & \multirow[t]{2}{*}{ Grupo D } & $\mathrm{f}$ & 56 & 21 & 29 & 2 & 108 \\
\hline & & $\%$ & $12,6 \%$ & $4,7 \%$ & $6,5 \%$ & $0,5 \%$ & $24,3 \%$ \\
\hline \multirow{2}{*}{\multicolumn{2}{|c|}{ Total }} & $\mathrm{f}$ & 223 & 102 & 109 & 10 & 444 \\
\hline & & $\%$ & $50,2 \%$ & $23,0 \%$ & $24,5 \%$ & $2,3 \%$ & $100,0 \%$ \\
\hline
\end{tabular}

Fuente: Los Autores

Al probar la hipótesis de independencia entre las variables categóricas por grupos y nivel de comprensión encontramos un valor de chicuadrado con 9 grados de libertad de 6,08 y un $\mathrm{p}$-valor asociado $(\mathrm{p}=0,7314>0,05)$, lo cual conduce a no rechazar la hipótesis de independencia y concluir que la discrepancia entre los niveles de comprensión no está asociada a un grupo específico.

\section{Conclusiones}

De lo observado anteriormente se puede concluir que las principales dificultades en la comprensión de gráficos estadísticos mostradas por los estudiantes de acuerdo con el modelo empleado en esta investigación son las siguientes:

Nivel idiosincrásico: Confunden los ejes, las unidades de los ejes y no identifican las unidades de medida de cada eje. Es el caso de las respuestas dadas a la pregunta ¿Cuántos días fueron observados para analizar la demanda del producto? Explique su respuesta. Los estudiantes asociaron equivocadamente el último ítem del eje y la frecuencia de los días con el total de días observados para la demanda, o asociaron la altura de la barra de mayor frecuencia con el total de días observados para la demanda.

En la pregunta: Sume las cantidades demandadas en 60 dias de observación, en donde hubo la mayor demanda del producto. Explique su respuesta, los estudiantes mostraron dificultades para identificar las unidades de medida de cada eje, asociaron la altura de la barra de mayor frecuencia con la frecuencia de días donde hubo mayor demanda o no identificaron los ítems del eje $\mathrm{x}$, cantidad de unidades demandadas, donde hubo la mayor demanda del producto y por consiguiente no identificaron apropiadamente los ítems específicos de la mayor demanda para luego sumarlos.

No identifican ningún rasgo significativo de la gráfica tanto a nivel cuantitativo como descriptivo. Cuando se les preguntó: Suma las cantidades demandadas en 60 dias de observación, en donde hubo la mayor
Jul - Dic 2016

ISSN 0122-820X

E-ISSN 2422-5053

PP: $13-23$ 
No. 2

Jul - Dic 2016 ISSN 0122-820X E-ISSN 2422-5053

PP: 13-23 demanda del producto. Explique su respuesta, simplemente escribieron un número cualquiera, la frase No sé o se limitaron a dejar en blanco el espacio correspondiente.

Nivel transicional: No utilizan etiquetas para identificar las variables expresadas en la gráfica, omiten las escalas en alguno de los ejes (horizontal o vertical) o en ambos, no especifican el origen de coordenadas ni proporcionan suficientes divisiones en las escalas de los ejes. Estas dificultades las tienen los estudiantes en el proceso de representación de datos, porque la carencia en el conocimiento de los elementos correspondientes a una gráfica impide la comprensión de relaciones entre los datos; los estudiantes se ubican en el nivel transicional porque para la pregunta: Complete la tabla de frecuencia que se presenta a continuación y bosqueje un diagrama de barras con los datos obtenidos, diseñaron inadecuadamente una escala, graduaron incorrectamente los ejes, no usaron etiquetas para identificar los objetos que se estaban graficando o construyeron una visualización incompleta de los datos.

Nivel cuantitativo: No justifican las respuestas. Esta dificultad la tienen los estudiantes dentro del proceso de descripción de datos, por ejemplo, con el gráfico diagrama de frecuencia y la pregunta $1 \mathrm{~A}$, inciso a) ¿Cuántos días fueron observado, en los que se demandaron 34 unidades?. La respuesta implicaba la capacidad para reconocer las unidades de medida de cada eje (en este caso, que el eje $x$ mide la cantidad de unidades demandadas, mientras el eje $y$ las frecuencias de dichas cantidades demandadas diariamente) y ser capaces de relacionar la cantidad de unidades demandadas con su frecuencia diaria, para de este modo "leer un dato". El error de los estudiantes estivo en colocar la cantidad correcta sin justificar su respuesta.
Nivel analítico: Este nivel no debería presentar para el estudiante universitario ninguna dificultad. Sin embargo, en este estudio fue el nivel menos productivo. Para futuras investigaciones, por tanto, es necesario y urgente diseñar actividades que permitan a los estudiantes un tránsito adecuado hacia niveles de comprensión superior.

Con respecto a la jerarquía en los niveles de comprensión se encontró que las tres cuartas partes de los estudiantes tienen serias dificultades para entender el contenido del gráfico y plantearse una posible lectura de interpretación, es decir, la gran mayoría se ubica en los niveles idiosincrático y transicional. Sólo una pequeña parte del grupo tiene una idea para plantearse una respuesta adecuada y un mínimo grupo es capaz de presentar una respuesta correcta.

El estudio identificó las principales dificultades que presentan los alumnos en la comprensión de gráficas. Tales dificultades permitieron ubicar a los estudiantes en los distintos niveles cognitivos de acuerdo con el marco propuesto por [9]. La identificación de estas dificultades y errores constituirán la base de futuras investigaciones destinadas al diseño de creativo y alternativo de actividades y recursos útiles para la conducción de los estudiantes hacia los planos más complejos de la comprensión. Tales actividades y recursos deben incluir no solo una perspectiva deductiva sino además una mejor conexión con la realidad (social, pero también cognitiva) de los estudiantes. En efecto, cuando los errores se encuentran en unos pocos estudiantes el problema es seguramente individual; pero cuando se hallan en la mayoría, el problema probablemente esté vinculado con la forma de enseñanza, con la perspectiva que se asume. Estas afirmaciones se encuentra en sintonía con la opinión de [7], quien sostiene que "es preciso intentar incorporar el enfoque heurístico y constructivo - frente al enfoque 
deductivista- en la presentación de los principales conceptos matemáticos a los alumnos de los primeros ciclos universitarios. Con frecuencia, en la metodología deductivista se presentan los distintos teoremas ya 'acabados' y 'perfectos'. Sin embargo, para llegar a cualquiera de estos resultados ha sido necesario, en primer lugar, su intuición, el comprobar para valores particulares cómo se cumple, y por último, tras varios intentos la demostración definitiva".

\section{Referencias}

[1] J. P. Arteaga, C. Batanero, G. Cañadas, and J. M. Contreras. "Las tablas y gráficos estadísticos como objetos culturales", Números, vol. 76, pp. 5567, marzo 2011.

[2] I. Cazorla. “A relação entre a habilidades viso-pictóricas e o dominio de conceitos estatísticos na leitura de gráficos", Tesis doctoral inédita, Universidad Estatal de Campinas, Brasil, 2002.

[3] C. Wild and M. Pfannkuch. "Statistical thinking in empirical enquiry". International statistical review, vol. 67, no. 3, pp. 223-265, 1999.

[4] S. N. Friel, G.W. Bright and F. Curcio. "Understanding Students Understanding of Graphs", Mathematics Teaching in the Middle School, vol. 3, no. 3, pp. 224-227, 1997.

[5] J. P. Arteaga, C. Batanero, J. Ortiz and J.M. Contreras. "Sentido numérico y gráficos estadísticos en la formación de profesores". Publicaciones, no. 41, pp. 33-49, 2011.

[6] J. D. Godino and C. Batanero, "Significado institucional y personal de los objetos matemáticos", Recherches en Didactique des Mathématiques, vol. 14, no. 3, pp. 325-355, 1994.
[7] J.P. Arteaga. "Evaluación de conocimientos sobre gráficos estadísticos y conocimientos didácticos de futuros profesores", Tesis doctoral, Universidad de Granada, Granada, 2011. [En línea]. Disponible en: http://www. ugr.es/ batanero/pages/ARTICULOS/ arteaga.pdf.

[8] J. M. Shaughnessy, J. Garfield and B. Greer. "Data Handling". En A. Bishop et al. (Ed.), International Handbook of mathematics education. Dordrecht: kluwer, A. P., vol. 1, 1996, pp. 205-237.

[9] C. W. Langrall and E. S. Mooney. "The development of a framework characterizing middle school student's statistical thinking". In B. Phillips (Ed), Proceedings of the sixth International conference on Teaching Statistics. Ciudad del Cabo, Sur Africa. 2002.

[10] R. Monroy Santana. "Categorización de la comprensión de gráficas estadísticas en estudiantes de secundaria (12-15)", REIEC, vol. 2, no. 2, 2007.

[11] F. Curcio. "Comprehension of mathematical relationships expressed in graphs", Journal for Research in Mathematics Education, vol. 18, no. 5, pp. 382-393, 1987.

[12] S. Friel, F. Curcio, and G. Bright. "Making sense of graphs: critical factors influencing comprehension and instructional implications". Journal for research in mathematics education, vol. 32, no. 2, pp. 124-158, 2001. 